\title{
A Numerical Thermal Analysis of the Heating Process of Large Size Forged Ingots
}

\author{
Nima Bohlooli Arkhazloo ${ }^{1, a^{*}}$, Farzad Bazdidi-Tehrani ${ }^{2, b}$, Morin Jean-Benoit ${ }^{3, c}$, \\ Mohammad Jahazi ${ }^{1, d}$
}

1Département de Génie Mécanique, École de Technologie Supériere, Montréal, H3C 1K3, Canada

${ }^{2} S c h o o l$ of Mechanical Engineering, Iran University of Science and Technology, Tehran 16846-13114, Iran

${ }^{3}$ Finkl Steel Inc., 100 McCarthy, Saint-Joseph-de-Sorel, QC J3R 3M8, Canada

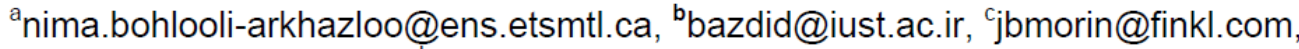
dmohammad.jahazi@etsmtl.ca

Keywords: CFD (Computational Fluid Dynamics), Heat treatment, Turbulent combustion, Cup Burner, Industrial gas-fired furnace, Transient heating 


\title{
A numerical thermal analysis of the heating process of large size forged ingots
}

\author{
Nima Bohlooli Arkhazloo ${ }^{1, a}$ *, Farzad Bazdidi-Tehrani' ${ }^{2, b}$, Morin Jean-Benoit ${ }^{3, c}$, \\ Mohammad Jahazi ${ }^{1, \mathrm{~d}}$ \\ ${ }^{1}$ Département de Génie Mécanique, École de Technologie Supériere, Montréal, H3C 1K3, Canada \\ ${ }^{2}$ School of Mechanical Engineering, Iran University of Science and Technology, Tehran \\ 16846-13114, Iran \\ ${ }^{3}$ Finkl Steel Inc., 100 McCarthy, Saint-Joseph-de-Sorel, QC J3R 3M8, Canada \\ anima.bohlooli-arkhazloo@ens.etsmtl.ca, ${ }^{\text {b }}$ bazdid@iust.ac.ir, ${ }^{c}$ jbmorin@ finkl.com, \\ ${ }^{\mathrm{d}}$ mohammad.jahazi@etsmtl.ca
}

\section{Keywords: CFD (Computational Fluid Dynamics), Heat treatment, Turbulent combustion, Cup Burner, Industrial gas-fired furnace, Transient heating}

\begin{abstract}
Simulation and analysis of thermal interactions during heat treatment is of great importance for accurate prediction of temperature evolution of work pieces and consequently controlling the final microstructure and mechanical properties of products. In the present study, a three-dimensional CFD model was employed to predict the heating process of large size forged ingots inside an industrial gas-fired heat treatment furnace. One-ninth section of a loaded furnace, including details such as fixing bars and high-momentum cup burners, was employed as the computational domain. The simulations were conducted using the ANSYS-FLUENT commercial CFD package. The k- $\epsilon, \mathrm{P}-1$ and Probability Density Function (PDF) in the non-premix combustion, as low computational cost numerical approaches were employed to simulate the turbulent fluid flow, thermal radiation, combustion and conjugate heat transfer inside the furnace. Temperature measurement at different locations of the forged ingot surfaces were used to validate the transient numerical simulations.
\end{abstract}

Good agreement was obtained between the predictions of the CFD model and the experimental measurements, demonstrating the reliability of the proposed approach and application of the model for process optimization purposes. Detailed analysis of conjugate heat transfer together with the turbulent combustion showed that the temperature evolution of the product was 
significantly dependant on the furnace geometry and the severity of turbulent flow structures in the furnace.

\section{Introduction}

Heat treatment, as a common practice in the steel making industry is an energy-intensive process with the largest portion of the consumed energy associated with the heating process of work pieces in the furnaces [1]. One of the major issues during the heat treatment process of large size parts, such as landing gears and turbine shafts is the control of temperature distribution inside the furnace and the product. Non-uniform temperature distribution may result in variation in mechanical properties and microstructure from one end to another of the part, increased distortion, or even scrapping of the part. Therefore, a better understanding of the thermal interactions is a critical parameter to assess the efficiency of the heat treatment process.

Among the different types of furnaces, gas-fired furnaces are commonly applied in applications with large size forged ingots [1]. The thermal schedule for the heating stage is generally determined by empirical relations [2]. However, these relations cannot cover all gas-fired furnace types and product configurations and could not be considered as an efficient and optimized method from material property or energy consumption point of views. Direct measurement and examination of the transient temperature distribution inside the furnace and the parts are exceedingly difficult, mainly due to their very large sizes. On the other hand, simultaneous existence of combustion (heat and mass transfer), turbulence and conjugate heat transfer (radiation, convection and conduction) in the gas-fired furnace medium and in the part make the analytical studies very difficult

Therefore, numerical simulation of the heating process in gas-fired heat treatment furnaces is of great importance for the prediction and control of the ultimate microstructure, properties of the work pieces, and reduction of energy consumption. Recently, Computational Fluid Dynamics (CFD) has been identified as an efficient tool to analyze, optimize and increase the performance of industrial furnaces [3]. However, due to the huge computational cost of transient simulation, in the 
case of complex 3D models of large size gas-fired furnaces, most of the studies have been devoted to laboratory scale furnaces with small pieces and low process time.

In the present study, a three-dimensional CFD model was developed to simulate and analyze the heating process and to examine the transient heating uniformity of large size forged ingots in a large size gas-fired furnace (see Fig. 1). The CFD model consists in a combination of one-ninth section of a loaded furnace including furnace details (such as burners and fixing bars) along with the numerical approaches with lower computational demand, namely, P-1 (for the radiation) and nonpremixed combustion. Analysis of transient temperature evolution data along with the heat and fluid flow interactions and their mutual interrelations will be discussed in the paper.

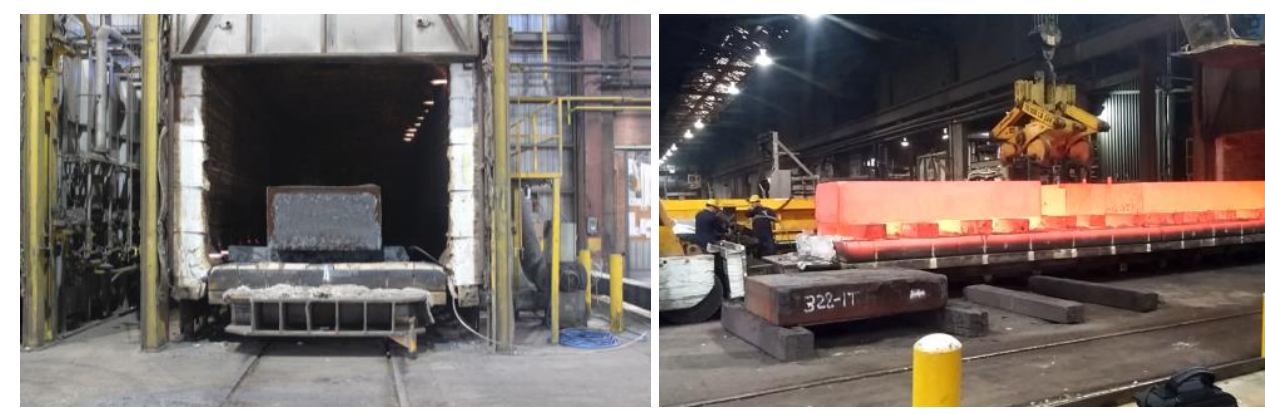

Fig. 1 Loaded gas-fired furnace at Finkel steel company [4]

\section{Furnace Description, Numerical Model and Boundary Conditions}

The present study was conducted on an industrial gas-fired heat treatment furnace located at Finkl steel, St-Joseph-de-Sorel, Quebec [4]. The furnace with internal dimensions of $3.05(\mathrm{~m}), 3.28(\mathrm{~m})$ and $8.33(\mathrm{~m})$ encompasses 18 high-velocity Tempest burners [5]. 


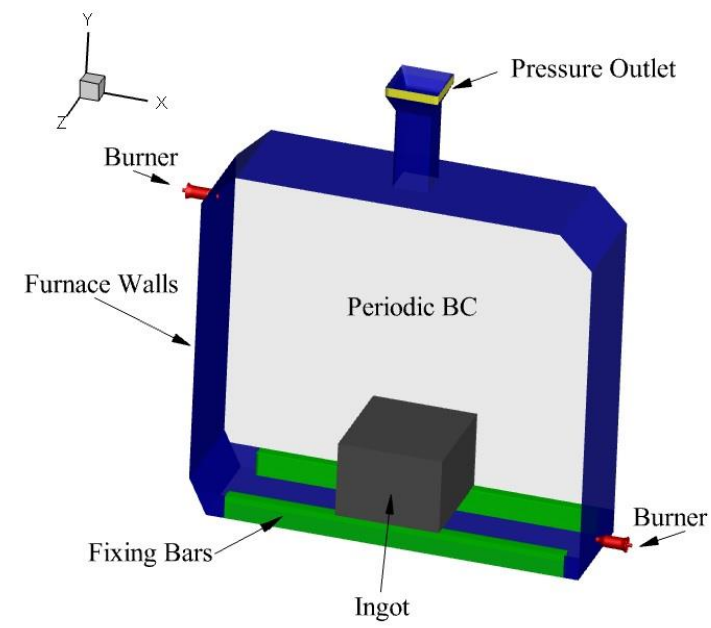

Fig.2 Proposed Numerical model and its relative boundary conditions

Burners were located in an asymmetric configuration, on two sides of the furnace (lower section of the right wall and upper section of the left wall). Temperature uniformity along the length of the furnace was assured by a PID system. Boundary conditions (BC) included mass flow inlet BC at the burners air and gas inlets and pressure outlet BC at the chimney exhaust. Model description, including applied BCs, is presented in Fig. 2. Complementary data about the general operation of the fully loaded furnace (in the $\mathrm{Z}$ direction with three large size blocs) are presented in Table 1.

Table 1. Operating data of the gas-fired furnace

\begin{tabular}{|c|c|}
\hline Item & Averaged value \\
\hline Furnace dimensions $(\mathrm{m})$ & $3.05 \times 3.28 \times 8.33$ \\
\hline Ingots dimensions $(\mathrm{m})$ & $1.17 \times 0.88 \times 2.74$ \\
\hline Fuel used & Natural Gas \\
\hline Tempering treatment temperature $(\mathrm{k})$ & 845 \\
\hline Average heat loss of furnace by side walls $\left(\mathrm{W} / \mathrm{m}^{2}\right)$ & 780 \\
\hline Gas mass flow rate $(\mathrm{kg} / \mathrm{s})$ & 0.0273 \\
\hline Air flow rate $(\mathrm{kg} / \mathrm{s})$ & 0.56056 \\
\hline
\end{tabular}




\section{Computational Details}

The solution to the general transport equations (continuity, momentum, and energy) was performed in the ANSYS-FLUENT [6] commercial software, using the finite volume method and applying the SIMPLE algorithm [6] to hexahedral non-uniform computational grids. Besides, the standard k- $\varepsilon$ [7] and P-1[8] models which have been reported to have good agreement with experimental observations in the literature of turbulent combustion applications [7,8] were employed for the turbulence and radiation modeling, respectively. The P-1 model specifically provides reliable results for the cases where the optical thickness, $(a+\sigma) \times L$, is larger than unity [9]. Optical thickness parameter encompasses the simultaneous effect of absorption, a, scattering, $\sigma$, and domain length scale L. Considering the size of the furnace, this model was employed to evaluate its possible application in the present investigation. The P-1 model [8] belongs to the general P-N model that is based on the expansion of the radiation intensity, I, into an orthogonal series of spherical harmonics. The P-1 model solves the radiation equation as a diffusion equation, which means a lower amount of CPU usage in comparison with other models such as the Discrete Ordinates (DO) model [9]. The other advantage of the P-1 model is its compatibility with complex geometries.

$$
\nabla .(\Gamma \nabla G)-a G+4 a \sigma T^{4}=S_{G} \quad \quad \Gamma=\frac{1}{\left(3\left(\mathrm{a}+\sigma_{s}\right)-C \sigma_{s}\right)}
$$

Natural gas combustion modeling using the Probability Density Function (PDF) in the non-premixed combustion model [10] was done in the present study. This model has also lower computational cost in comparison to the conventional models such as Eddy Dissipation model (EDM) [6].

\section{Experimental Measurements}

To assess the proposed approach and validate the simulation results three forged ingots with the dimensions of $1.45 \times 0.88 \times 2.75(\mathrm{~m})$ were positioned along the length of the furnace in the $\mathrm{Z}$ direction and at equal distances from the side walls. Three thermocouples, at the center of the right, up and left surfaces of the center forged ingot, called TC-1, TC-2 and TC-3 respectively, were attached to capture 
temperature evolution during the heating process. The chemical composition of the alloys is presented in Table 2. A layer of oxide covered the surface of the blocs before the start of the heating process.

Table 2: Chemical analysis of the forged ingots - \% Weight [4]

\begin{tabular}{|c|c|c|c|c|c|c|}
\hline $\mathrm{C}$ & $\mathrm{Mn}$ & $\mathrm{Si}$ & $\mathrm{Mo}$ & $\mathrm{Cr}$ & $\mathrm{Ni}$ & Other \\
\hline 0.35 & 0.85 & 0.4 & 0.45 & 1.85 & 0.47 & Micro alloying \\
\hline
\end{tabular}

\section{Results and Discussion}

Fig. 3 represents a comparison of the predicted and measured temperatures of the forged ingot during the process.

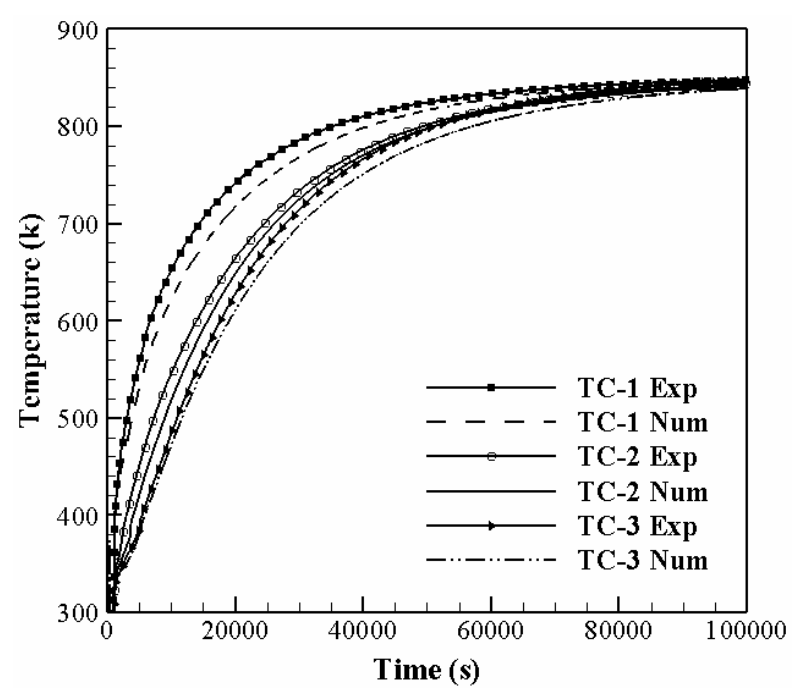

Fig. 3 Comparison of predicted and measured temperatures at the right (TC-1), up (TC-2) and left (TC-3) surfaces of the forged ingot

Results indicate that during the heating process the forged ingot had higher heating rates at the beginning of the process (sharp temperature differences), while the heating rate reduced near the target soaking temperature. Results also show a temperature difference of up to $200 \mathrm{~K}$ between different points of the forged ingot, specifically between those of the right (TC-1) and the left (TC3) surface. This non-uniform heating pattern could be due to the burner location and fluid flow interactions inside the furnace. Fig. 3 demonstrates that the non-uniformity reduced at the end of the 
process where the heating rate reduced and all the TCs converged to the target temperature. Besides, it can be seen that there is good agreement between the results of TC-2 and TC-3; whilst an underprediction relative to the experiment can be found in the results of TC-1. Considering the fact that TC-1 was facing the burner and its temperature is sensitive to the exact position of the thermocouple, the prediction could be considered fairly well. Therefore, usage of the P-1 model in the large size furnace where the optical thickness, $(\mathrm{a}+\sigma) \times \mathrm{L}$, due to the length scale of the furnace, $\mathrm{L}$, is larger than unity can help to save the computational time while keeping reasonable accuracy.

On the other hand, although Tempest burners are characterized by about $20 \%$ premix condition, the non-premix combustion model used in the present investigation provides good predictions. Therefore, the non-premix model can be used instead of the partially-premix model when the heat transfer to the product is the main objective rather than combustion phenomena. The main benefits of using the non-premix combustion model are reduced complexity of convergence and lower computational cost. Therefore, it can be employed in this application, where the size of the furnace and the number of grids are the main constraints for the simulations.

Transient temperature contours of the forged bloc during the process are presented in Fig. 4. A non-uniform temperature distribution on different surfaces of the product can be seen in this Figure. Specifically the temperature difference between the right wall, which is directly faced with the burner, and the left wall, is evident. The minimum transient temperature was predicted for the upper side of the left wall. Such a non-uniform heating for many hours can cause a significant change in the microstructure evolution in the part on different surfaces and should be taken into consideration for the optimization purpose. The first reason for the observed non-uniformity is probably the location of the burners in the furnace. As it can be seen, the highest temperatures are predicted in the lower side of the right wall, which is closer to the burner, in comparison to the other parts. In contrast, the left surface, facing the furnace wall, has lower temperatures. Therefore, the geometrical effect of the furnace and the relative location of forged ingots inside the furnace can 
change the heating pattern and should be considered for accurate quality assessment of the heating process.

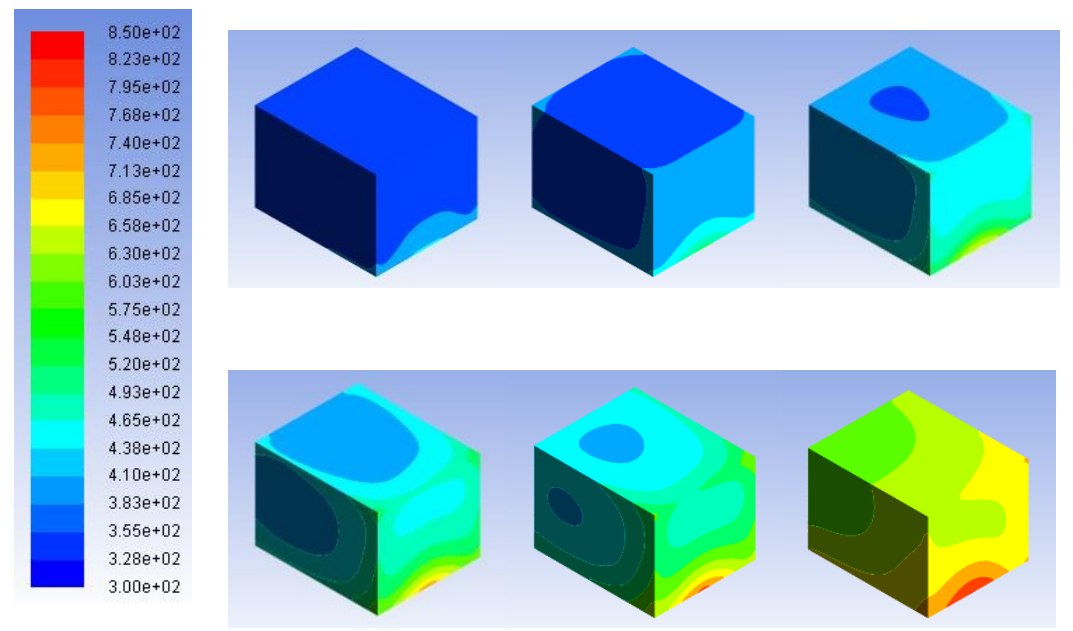

Fig. 4 Transient contours of temperature evolution of the forged ingot surfaces

The other possible reason for this non-uniformity could be tracked by examining the velocity vectors and streamlines of the furnace flow field as depicted in Fig. 5.

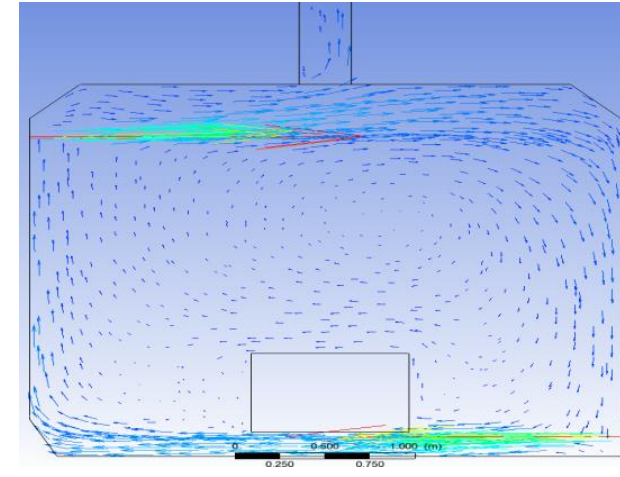

(a)

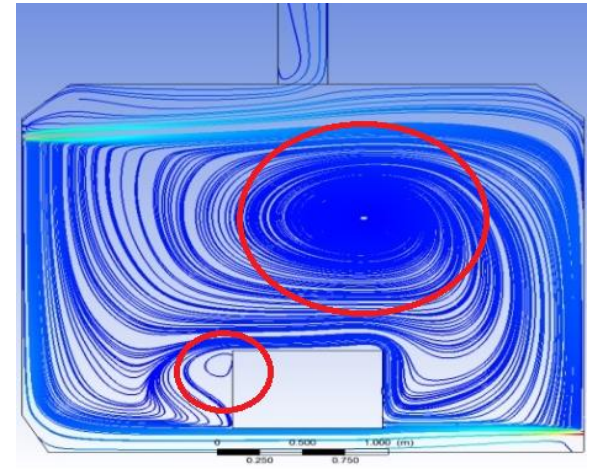

(b)

Fig.5 (a): Velocity vector and (b): time averaged streamlines of the loaded furnace

Counter clockwise circulation of flow in the furnace as well as blockage of flow (in other words blockage of heat and fluid flow) is evident. Therefore, the right wall blocking the fluid flow circulation absorbs more energy at the stagnation point and its surrounding. Besides, the local heat transfer to the part is also dependent on the formation of vortical structures or vortices around it [11]. One of the main characteristics of the vortical structures is making a coherent circulation in 
parts of the flow field [11]. Hence, vortical structure can be recognized using streamlines and velocity vectors. In the present investigation a small vortex in the wake region of the forged ingot (near the left wall) and a large and strong one in the center of the furnace were revealed by the CFD model as shown in Fig. 5. These findings indicate that, a correlation can be made between vortical structures and the heating pattern in the furnace. Specifically, the coexistence of a vortex in the wake region of the forged ingot and a lower temperature in the vicinity of the product walls proves the existence of this inter-relation, which can be used for optimization purposes in multiple loading configurations.

\section{Summary}

In the present study, a 3-D transient CFD model combining the one-ninth section of an industrial gas-fired heat treatment furnace was developed and proposed for the analysis of the heating process of large size forged ingots in these furnaces. Low computational demand approaches for the radiation and combustion simulation, p-1 and non-premix combustion were used in the model. The Following conclusions can be made from the present study:

1. The developed CFD model was proved to predict the measured temperature in the real process and the proposed combination could be used for the optimization purposes.

2. P-1 model characterized by a lower computational cost in comparison to the conventional models such as EDM could be used as a reliable approach for simulating the radiation in large size gas-fired furnaces.

3. Non-premix combustion modeling, could be validated alongside the other parts of the proposed model for this application. This model, even for the cases with partly partiallypremix nature, can be used in the industrial applications, where the analysis of the product heating is the major objective. 
4. Furnace geometry and vortical structures in the furnace flow field can be correlated with the heating pattern of the product and should be considered for the optimization of multiple loading patterns.

\section{Acknowledgement}

Financial support from MITACS in the framework of the IT03151 Grant is acknowledged. The authors are very grateful to Finkl Steel, especially the R \& D, Metallurgy and Engineering Departments, for providing the large size bloc, as well as the instrumentation and measurements used in the present research.

\section{References}

1. Kang, Jinwu, and Yiming Rong., Modeling and simulation of load heating in heat treatment furnaces." Journal of materials processing technology, 174.1-3, (2006): pp. 109-114.

2. Gao, M., et al., Estimating equilibration times and heating/cooling rates in heat treatment of workpieces with arbitrary geometry. Journal of materials engineering and performance, 9(1) (2000), pp. 62-71.

3. Mayr, B., et al., CFD analysis of a pusher type reheating furnace and the billet heating characteristic. Applied Thermal Engineering, 115 (2017), pp. 986-994.

4. $\quad$ Finkl Steel Inc., M., Saint-Joseph-de-Sorel, QC J3R 3M8, Canada.

5. Baukal Jr, C.E., Industrial burners handbook. 2003: CRC press.

6. ANSYS Fluent Theory Guide 13.0, ANSYS, Inc, Canonsburg, PA, USA, 2010.

7. Liu, Y. J., et al., A numerical analysis of slab heating characteristics in a rolling type reheating furnace with pulse combustion, Applied Thermal Engineering, 107 (2016), pp. 1304-1312. 
8. Hachem, Elie, et al., Modeling of heat transfer and turbulent flows inside industrial furnaces., Simulation Modelling Practice and Theory, 13 (2013), pp. 35-53.

9. Porter, R., et al., Evaluation of solution methods for radiative heat transfer in gaseous oxyfuel combustion environments., Journal of Quantitative Spectroscopy and Radiative Transfer, 111.14 (2010), pp. 2084-2094.

10. Zhang, C., et al., The numerical and experimental study of non-premixed combustion flames in regenerative furnaces., Journal of Heat Transfer, 122.2 (2000), pp. 287-293.

11. Ničeno, B., A. Dronkers, and K. Hanjalić, Turbulent heat transfer from a multi-layered wallmounted cube matrix: a large eddy simulation. International Journal of Heat and fluid flow, 23 (2002), pp. 173-185. 